\title{
The Impact of Supercomputing Capabilities on U.S. Materials Science and Technology *
}

\author{
William D. WILSON \\ Computation Department, Sandia National Laboratories, \\ Livermore, California, U.S.A.
}

Robert J. ASARO

Division of Engineering, Brown University, U.S.A.

\section{Robert W. DUTTON}

Center for Integrated Systems, Stanford University, U.S.A.

\section{Juan M. SANCHEZ \\ Henry Krumb School of Mines, Columbia University, U.S.A.}

\section{David J. SROLOVITZ}

Department of Materials Science and Engineering, University of Michigan, U.S.A.

\section{Richard H. BOYD}

Department of Materials Science and Engineering, University of Utah, U.S.A

William A. GODDARD, III

Arthur Amos Noyes Laboratory of Chemical Physics, California Institute of Technology, U.S.A.

\section{John R. SMITH}

Physics Department, General Motors Research Laboratories, U.S.A.

\section{Wilhelm G. WOLFER}

Physical Science Department, Sandia National Laboratories, Livermore, California, U.S.A.

A committee was formed under the auspices of the National Academy of Sciences to identify areas

* This paper was originally published as part of a report by the authors as members of the Committee on Computer Simulation and Analysis of Complex Material Phenomena. It is reprinted here with permission of the National Materials Advisory Board, Commission on Engineering and Technical Systems. National Research Council.

\footnotetext{
North-Holland

Future Generation Computer Systems 5 (1989) 283-293
}

of materials science and engineering where a major impact might be realized, resulting from the emergence of supercomputer technology. A great number of examples of exciting individual computational science were identified: Atomistic and electronic structure calculations on metals, semiconductors, and polymers; statistical mechanical studies of alloy phase diagrams; and fundamental modeling of fracture and deformation in metals, ceramics, and glasses are among those that are vigorously exploiting supercomputer technology. Moreover, electronic structure effects on a scale of angstroms are being coupled with microstructural aspects on a scale of micrometers, and these are further coupled to continuum effects on a scale of centimeters. Supercomputers are emerging as powerful and cost-effective tools, not only for the furtherance of materials science, but also for linking this science with engineering, design, and manufacturing. In this paper each example stands alone, consisting of a brief technological background followed by the specific supercomputer examples.

\section{Executise Summary}

The primary task of this study was to identify key opportunities that would make a major impact in materials science and engineering, exploiting the emerging capabilities afforded by large-scale supercomputers. The committee applied rigorous criteria: The equations or algorithms should be known well enough that progress can be demonstrated today and the future reasonably and credibly predicted. Examples of how "given a computer ten times faster, it will be possible to..." were discarded if not preceded by "we have shown, in agreement with experiment, that..."

Our committee was selected to represent universities, industry, and national laboratories from a broad geographical base and a wide variety of disciplines. We began by bringing together, as an intense workshop in San Diego (March 21-24, 1987), experts from the wide variety of disciplines comprising materials science and engineering (materials scientists, physicists, chemists, metal- 
lurgists, engineers, polymer scientists, etc.). Papers were solicited before the meeting, presentations were made, and follow-up discussions pursued. The vast amount of material collected was reduced to a manageable level at a committee meeting in July 1987.

We found early on that the computational capability of supercomputers (the largest and most powerful computers in existence at any time) is continuing to increase rapidly at roughly constant cost. Cray Research has announced the Cray-3; ETA, Inc., the ETA-10. NEC and Fujitsu appear committed to becoming the world's supercomputer manufacturers, utilizing parallel processors to achieve their performance goals. Simultaneously, massively parallel processing machines are being designed that are capable of unlimited growth in computing power. It is clear that for the first time in history a tool has emerged that can aid in understanding materials processes at the level of complexity required to make an impact.

We made a strong distinction between the $c a$ pability of supercomputers and the capacity that can be gained by less expensive mainframes and workstations. We recognized that a hierarchy of computing architectures needs to be employed (e.g. workstations are more efficiently used for graphics than mainframes are), but we restricted ourselves to the kinds of problems that cannot be done any other way. The scientific and engineering complications are so great that, in many cases, computation is the method of choice over experiment.

We found molecular mechanics calculations on polymers being performed that could predict the elastic properties of a complex polypropylene glass or the heat capacity of polyoxymethylene. Such calculations are ultimately capable of predicting the constitutive laws necessary to design polymerforming dies by computer, with enormous cost savings.

We found that supercomputers have become powerful enough to predict phase diagrams of simple alloys from first principles, in agreement with experiment, giving the historic field of alloy development its first real hope of designing alloys by computer. Although it is not possible to design complex alloys by computer today, the economic impact of being able to design specialty alloys by computer for a declining steel industry is large enough to drive continuing work in this area.
The economic advantages of silicon semiconductor technology are being exploited by growing layers of germanium on silicon substrates, a nonequilibrium thermodynamic process now capable of being understood computationally. The layer thickness at which deleterious misfit dislocations are introduced in the growth process can be predicted by supercomputer. "Band gap engineering" - the ability to tailor the band gap of a semiconductor to technological needs - is within reach. Already electron localization effects in superlattices can be predicted from first principles, in agreement with experiment.

An important first step has been made in understanding the complexities of adhesion, a problem that permeates nearly all manufacturing processes. A universal adhesive energy relation was discovered by extensive computation of the energy required to separate metallic surfaces. Surface science and engineering is at the heart of many present and future technologies (e.g. semiconductor interfaces and corrosion) and is particularly ripe for computation. Quantum chemical ( $a b$ initio) and solid state (local density functional) techniques have impressive successes to report.

One area that Japan dominates is the manufacture of the equipment for producing semiconductors. We found that supercomputing has already been successfully applied by U.S. researchers to the design of chemical vapor deposition equipment, specifically to the rotating disk reactor. Efforts such as this could enable the United States to regain its processing advantage.

Fundamental deformation and fracture mechanisms in metals, alloys, and ceramics are being studied extensively by supercomputing. The ability to describe the complex deformations of metals can lead to dramatic improvements in the design of forming processes. The atomistics of dislocation generation at a crack tip, including the effects of impurity atoms such as hydrogen ("hydrogen embrittlement"), can be calculated using realistic interactions between the atoms. Continuum models of microcrack density and propagation have reached a stage where numerical simulations can play a major role in design and performance assessments of, for example, though new ceramics. The supercomputer will make it possible to link methods such as these, thereby significantly changing the way materials design is carried out.

Pharmaceutical design, although not tradition- 
ally a materials problem, is becoming progressively more supercomputer-oriented because of the costs and risks associated with human experimentation. Lest it becomes lost, one example of the enormous strides being made in the design of chemotherapeutic drugs by supercomputer has been included in this report.

The list of examples here is by no means exhaustive: We found ourselves "stopping the presses" a number of times as still another paradigm of supercomputing in materials science came to our attention, and we recognize that some areas of computational materials science and engineering may not be covered. Yet we felt convinced by the number and depth of examples to come to several conclusions:

1. Supercomputer simulation will serve as a powerful and cost-effective link between materials science and engineering, design, and manufacturing.

2. New theoretical methods, algorithms, and approaches for analyzing the individual components of complex materials systems are rapidly evolving.

3. To exploit opportunities in computational materials science and engineering, the United States must invest heavily in the science underlying the methodologies, in developing software to make these methodologies useful to engineers and technicians, and in providing adequate hardware to scientists and engineers at universities and in industry.

4. To remain a leader as materials simulations moves out of the laboratory and into the industrial plant, the United States must act decisively now to stimulate the development of these methodologies and to develop turnkey simulation systems for industrial plants.

5. Simulations can reduce design costs and the time from concept to market.

6. Supercomputers allow the use of hierarchical design tools.

7. Supercomputer simulation is a powerful design tool that can increase U.S. economic competitiveness today.

Based on these conclusions, the committee has the following recommendations:

1. Focused multidisciplinary research initiatives in selected areas of materials science and engineering should be started in order to integrate design and manufacturing with the underpinning research base.
2. A Supercomputing Center for Materials Applications should be created. Such a center would provide a better means for conducting pioneering research and moving the results of basic research into turnkey systems suitable for wide use in U.S. industry.

3. Existing supercomputer centers must be kept at the state of the art.

4. Multidisciplinary materials simulation groups should be formed.

5. The infrastructure of local computing associated with advanced simulation must be strengthened.

6. These initiatives should be implemented in a manner balanced to preserve the research strengths that are central to the success of materials engineering and processing in the future.

Chemical Vapor Deposition Is ( ritical To Semiconductor Manufacturing

Chemical vapor deposition (CVD) is an increasingly important process in the United States and other countries for fabricating electronic components. Much of the emphasis on CVD in the microelectronics industry stems from the fact that it is well suited to producing sillicon films, which are essential in high-performance chips.

The process begins by passing an active gas, such as silane $\left(\mathrm{SiH}_{4}\right)$, over a substrate maintained at a temperature on the order of $1000 \mathrm{~K}$. As the heat is transported away from the substrate by convection, diffusion, and radiation, the active gas chemically decomposes to form species that are very reactive on the substrate itself. Upon reaction of the substrate, a solid (for example, silicon) is deposited and a volatile product (e.g. $\left.\mathrm{H}_{2}\right)$ is released back into the gas. Although this process is employed in semiconductor production today, the understanding of the process is still incomplete. Many reactors are designed by empirical rules and, as a result, are notoriously unreliable.

Important issues in the fabrication of semiconductors with CVD are deposition rate, deposition uniformity, and sharpness of interfaces between adjacent layers in compound semiconductors. A designer can control the reactor geometry, the flow rates of gases through the reactor, the low rate distributions at the inlet of the reactor, the rotation rate of the heated substrate in the case of 


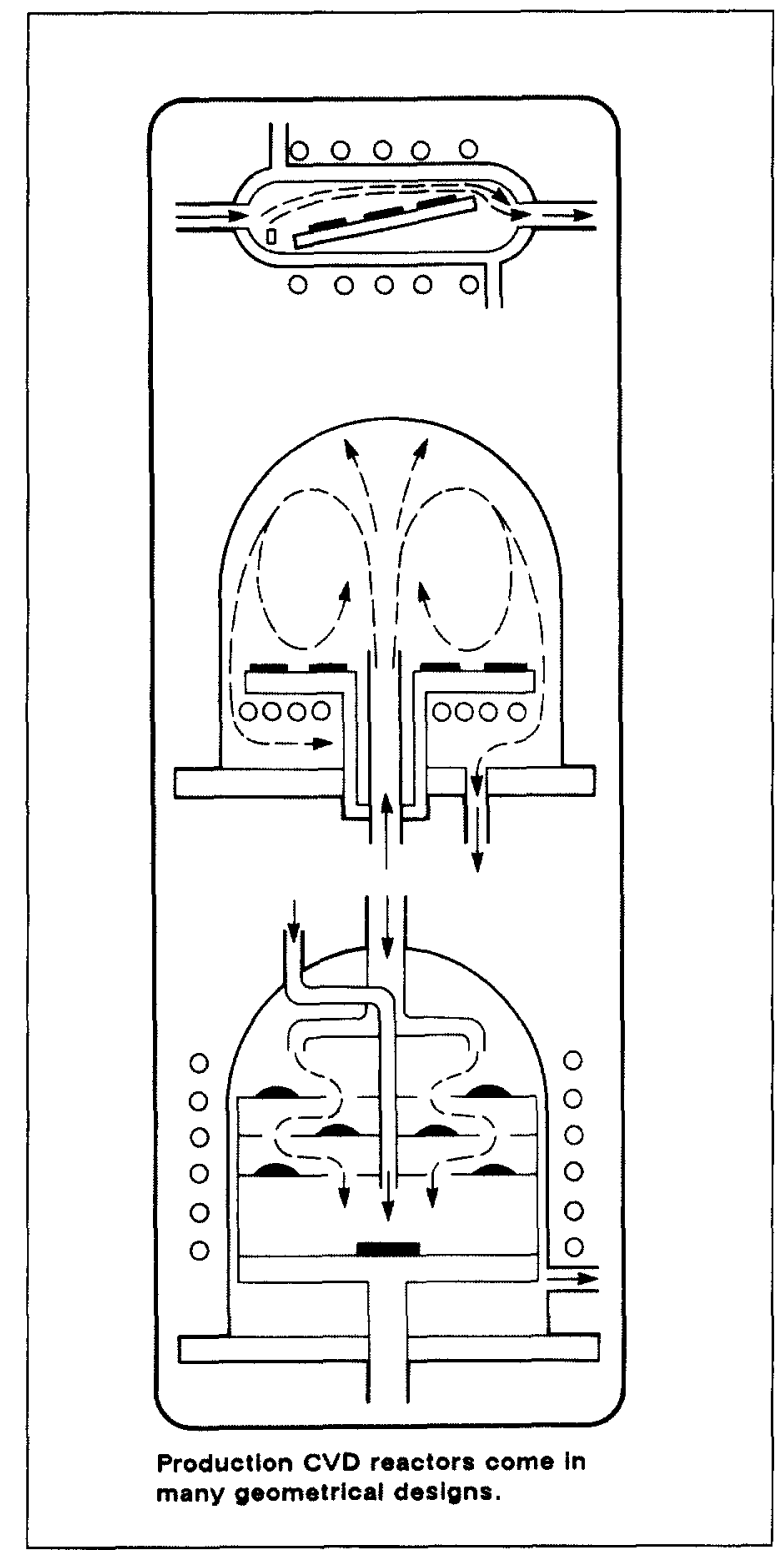

Fig. 1. Production CVD reactors come in many geometrical designs.

a rotating disk reactor, the thermal boundary conditions on the walls of the reactor, the temperature of the substrate and the reactant gas mixture and pressure. Production reactors employ a variety of geometrical design (as illustrated in Fig. 1 and Fig. 2), further complicating attempts at understanding this important materials process.
Computer Simulation Is Guiding Chemical Vapor Deposition Reactor Design

A multidisciplinary effort involving quantum chemistry, chemical kinetics, heat transfer, fluid dynamics, numerical analysis, and computer science is focusing on chemical vapor deposition. The effort is an outgrowth or research on combustion chemistry in which flow reactors, premixed flames, and diffusion flames are simulated. Common to all of these are large systems of gas-phase chemical reactions and complex molecular transport.

The simulation requires, as input, detailed thermodynamic properties for all the reacting species, including the source gases and all intermediate and product gases. Often the thermodynamic properties are not accessible by experiment, but state-of-the-art quantum chemical computational techniques can determine properties accurately. Rate expressions are also needed for all reaction paths, in the gas phase as well as on the surface. Although the most important rate constants must be measured experimentally, rate determinations can often be difficult. Fortunately, statistical reaction rate theory provides the tools to make accurate estimates.

Once the chemical reaction mechanism is established, conservation principles are used to derive equations that describe the complex interplay of fluid and heat transport with the chemical processes. The exact form of these large systems of partial differential equations depends on the particular reactor configuration. Typically, the system of equations is "stiff" (meaning that some of the chemical reactions are very rapid, while others are very slow). Such a system requires sophisticated mathematical techniques for its solution.

Initially this elaborate simulation was applied to the horizontal channel reactor (see above) where the importance of gas-phase chemistry was first demonstrated and later confirmed by experiment. More recently a rotating-disk reactor was modeled (Figs. 3 and 4). The heated substrate rotates at $1000 \mathrm{rpm}$ in a cylindrical shroud through which the reactive gases flow. The advantage is that the species and temperature gradients normal to the disk can be made to yield a highly uniform deposition. The simulations show that, at low temperatures, the rate of deposition of $\mathrm{Si}$ from $\mathrm{SiH}_{4}$ is determined by the rate of reaction of $\mathrm{SiH}_{4}$ on the 


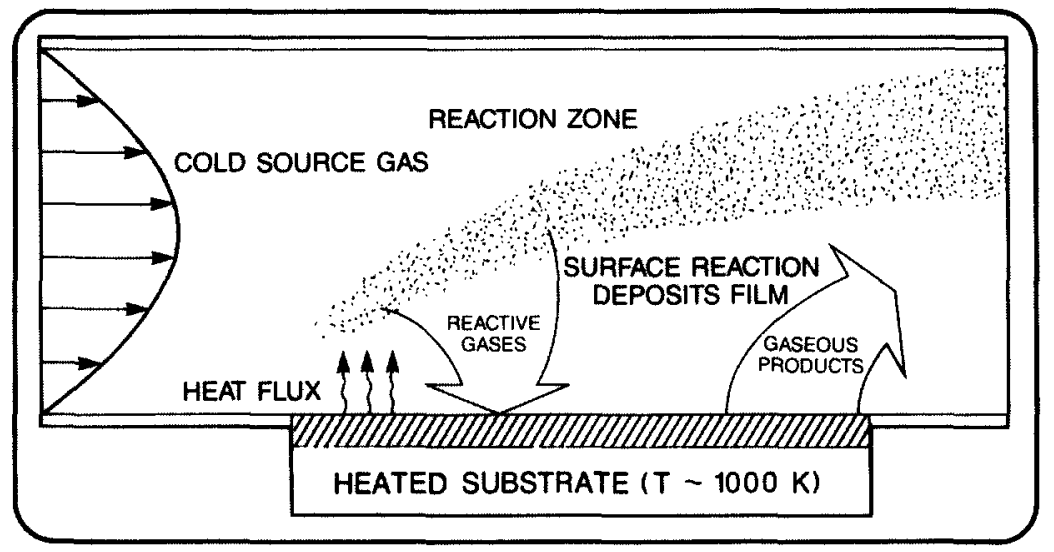

In this schematic of a horizontal channel CVD reactor, cold Inlet gas (such as

$\mathrm{SIH}_{4}$ ) reacts in the zone above a heated inert material (referred to in industry as the susceptor). Surface reactions result In the deposition of sillicon and the production of volatlle gases, which exit to the right.

Fig. 2. In this schematic of a horizontal channel CVD reactor, a cold inlet gas (such as $\left.\mathrm{SiH}_{4}\right)$ reacts in the zone above a heated inert material (referred to in industry as the susceptor). Surface reactions result in the deposition of silicon and the production of volatile gases, which exit to the right.

rotating surface. At intermediate temperatures the rate-limiting step is the gas-phase chemical kinetics, and at high temperatures it is diffusion. The effect of disk rotation on the rate of deposition was shown to depend on the process controlling the deposition.

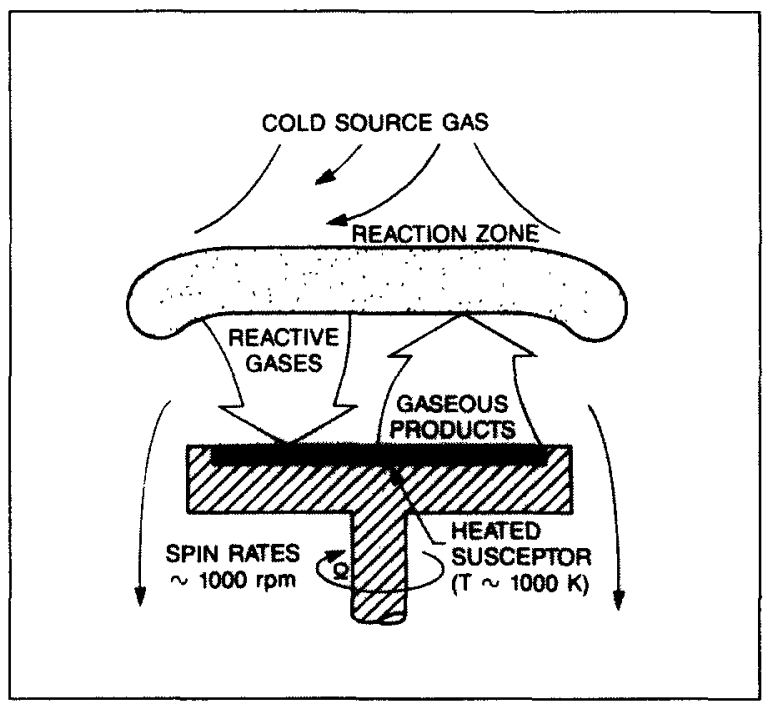

Fig. 3. The fluid flow and heat transfer in a rotating disk CVD reactor have been simulated and used by industry to guide growth conditions and reactor design.
These simulations have had a substantial impact on commercial reactor design through industrial interactions. The simulations are being used to guide the choice of temperature, total flow, pressure, and rotation speed in a production reactor. They would not be possible without a

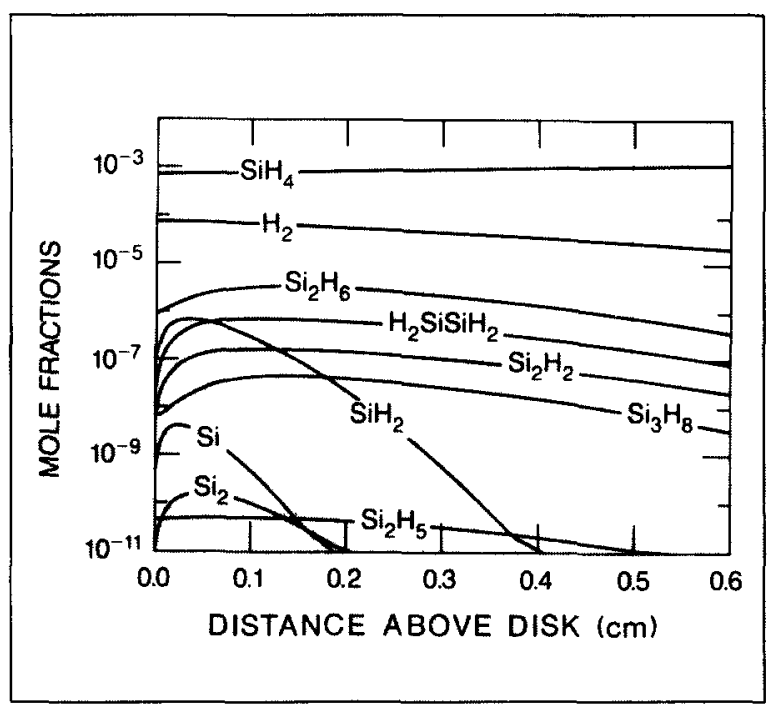

Fig. 4. Predicted density profiles of various chemical species as functions of height above the spinning disk. 
large number of supercomputer calculations, ranging from the solution of basic quantum phenomena to the detailed solution of macroscopic transport.

In the future, greater supercomputer capabilities will permit the chemistry and fluid flow to be even more closely coupled in CVD simulations. Other semiconductor materials processes, such as plasma etching, will also be simulated; and other processing concerns, such as the flow of particulate contaminants, will be accessible to simulation through hierarchical modeling.

Increased Complexity Is Driving Device Physics Toward Supercomputing

The continued growth of the electronics industry depends on a continued increase in packing density (number of components on a chip) so that electrons can travel over shorter and shorter distances. This trend in device miniaturization leads to inherently three-dimensional characteristics and to quantum behavior of electron transport in semiconductor materials (Fig. 5). The development of a new semiconductor chip is greatly complicated by these effects; each design iteration can cost hundreds of thousands of dollars and take months for engineers to learn the effect of design parameter variations.
Fortunately, electron transport calculations in semiconductors have evolved to a level of sophistication sufficient to be of value to the device engineer. The area of transport analysis has been - greatly affected by the numerical techniques of modern physics. Modern Monte Carlo simulations now incorporate realistic descriptions of the relationship between an electron's energy and its momentum (the "band structure" of the solid) instead of resorting to adjustable parameters to calculate electron transport properties in bulk materials. Furthermore, the scattering (deflection) of an electron by the vibrating atoms of the solid (phonons) and the ionized impurities in the solid can be meaningfully included. The results of these simulations are in excellent agreement with experiment and have been useful in understanding the physics of fast electrons in semiconductors (hot carriers). More recently, Monte Carlo techniques have been used in the area of real space electron transfer in order to model a number of electronic devices (see example in the next section).

Today, supercomputer simulation can make a major economic impact on semiconductor device design. As the scale of device structures decreases, however, the physical methodology must include a more quantum description of the electron transport (Fig. 5). Current studies are limited to quantum calculations on simplified model systems. The inclusion of fully quantum techniques in a device

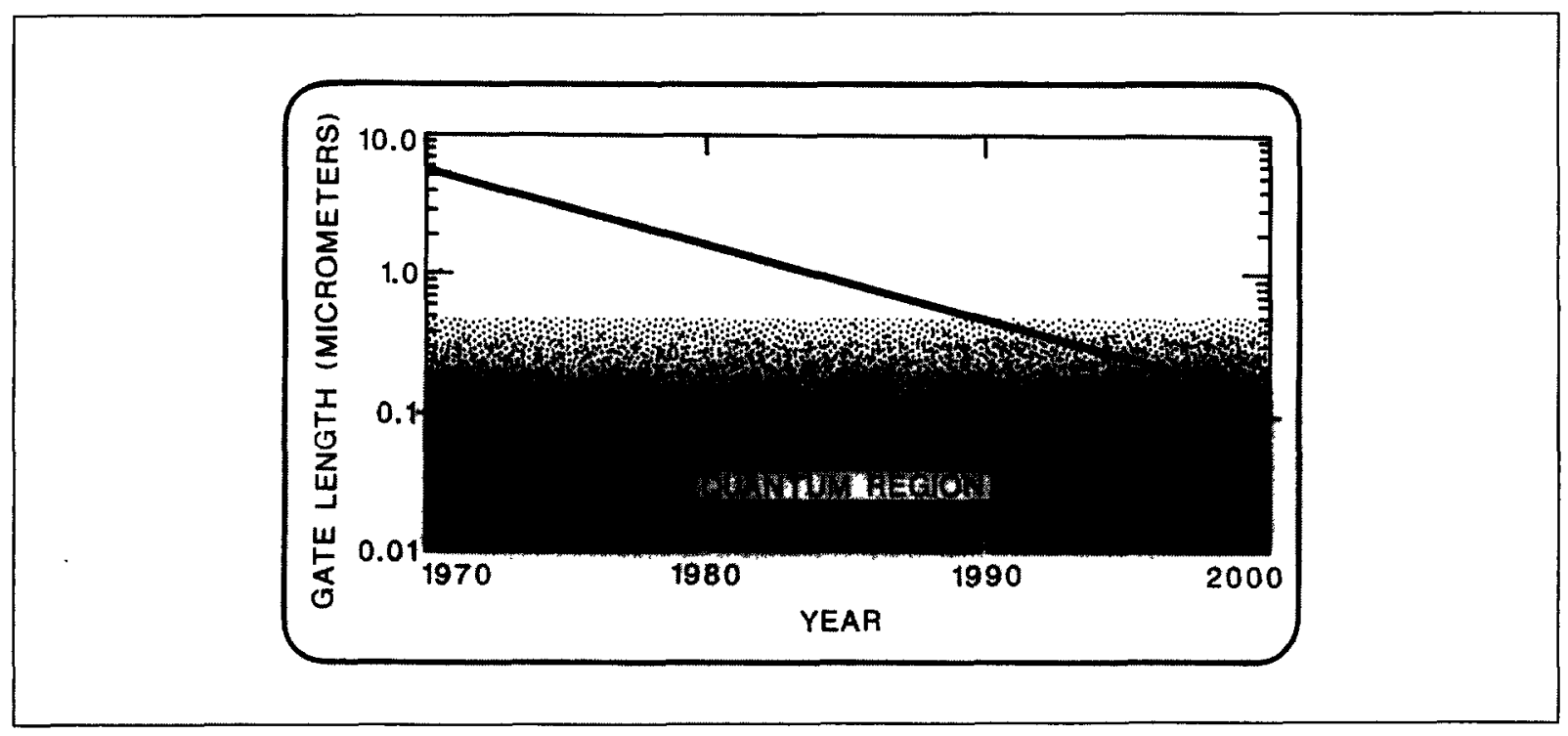

Fig. 5. Device sizes are approaching the quantum length scale. 
modeling code will require not only an increase in computer power but also improvements in the basic physics of time-dependent phenomena.

Revolutionary Desices Can Be Realistically Modeled

With recent advances in semiconductor device simulation it is now possible to assess the advantages of novel device structures before incurring hardware development time and costs. The velocity-modulated transistor concept provides an example.

In a typical semiconductor switch, electrons flow from the source (at zero potential) to the drain (biased positive) while the gate is biased positive (see Fig. 6). When the gate is switched to zero, electrons no longer flow; the characteristic switching time is that required for the electrons under the gate to travel a distance of order $D(0.5$ $\mu \mathrm{m})$ to the drain. Device designers requiring ultrafast switching times have proposed a velocitymodulated transistor (VMT), which capitalizes on the extremely short perpendicular transit times between two adjacent channels separated by a much shorter distance, $d$.

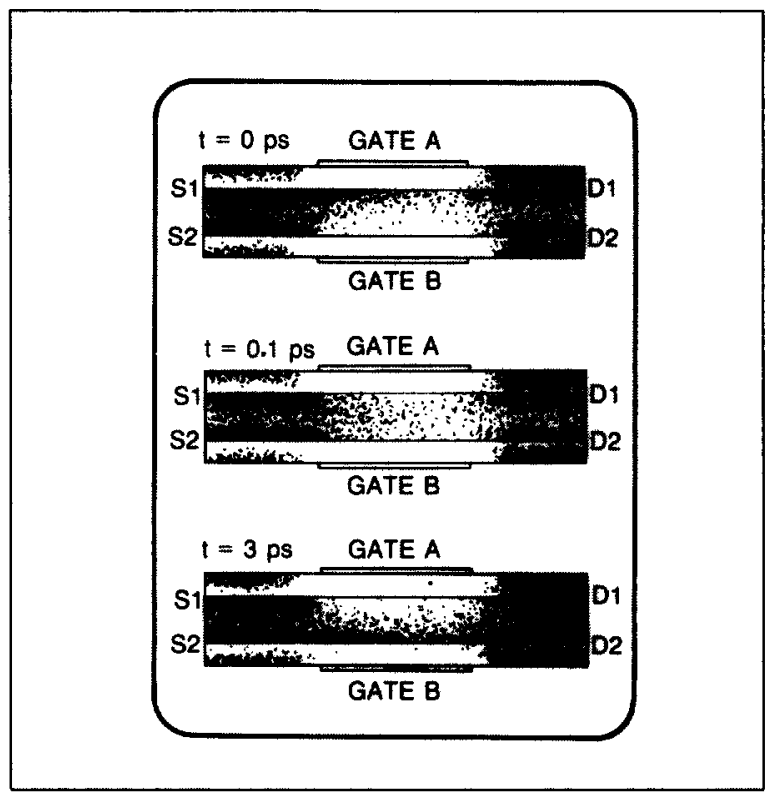

Fig. 7. Electron transfer from channel $A$ to $B$ in a VMT from 0 to 3 picosec. Electron density is indicated by the degree of darkness in each plot.

The VMT concept involves switching from a state with both sources at $0 \mathrm{~V}$, both drains at $+0.3 \mathrm{~V}$, and both gates at $+0.8 \mathrm{~V}$. At $t=0$, gate $\mathrm{A}$ is switched to 0 and gate $\mathrm{B}$ to $0.6 \mathrm{~V}$. Electrons

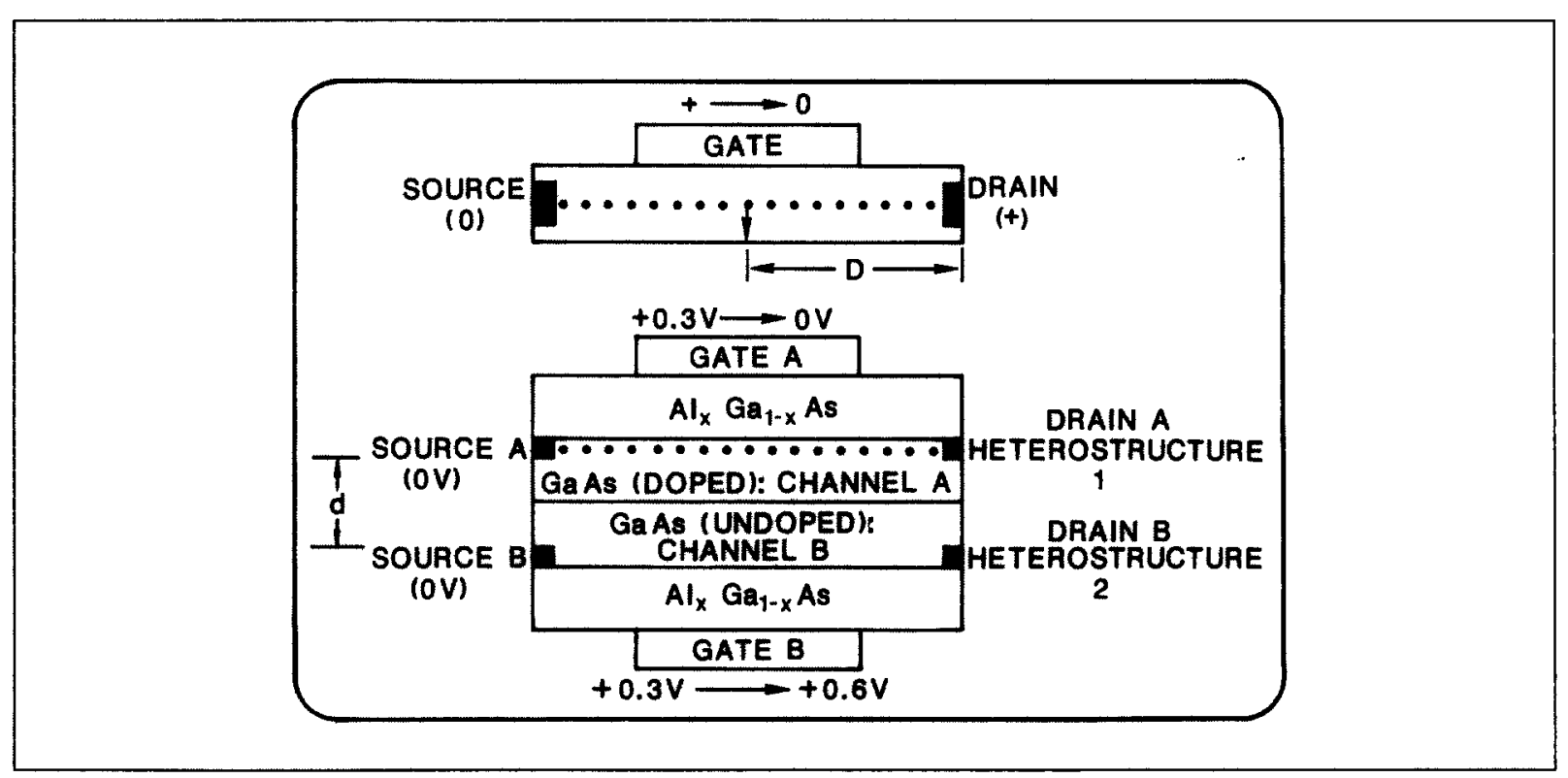

Fig. 6. Electrons in a traditional transistor (top) must travel a distance $D$ to turn the device off, whereas in a VMT, electrons need only travel a much shorter distance $d$. 
flowing in channel A (GaAs, doped region) are suddenly accelerated to gate $\mathrm{B}$, an order-of-magnitude smaller distance than that for a traditional transistor switch. Such a design involves optimizing such parameters as the $\mathrm{Al}_{x} \mathrm{Ga}_{1-x}$ alloy composition, degree of doping of the GaAs channel A, heterostructural thicknesses, geometry, and operating temperature.

The results of the Monte Carlo simulation of this device are shown in Fig. 7. The simulation includes complete band structures for the $\mathrm{GaAs}$ and $\mathrm{AlGaAs}$ regions and the effects of all physically important scattering mechanisms (i.e. polar optical phonon scattering, equivalent and nonequivalent intervalley scattering, ionized impurity scattering, and impact ionization). The dark areas show how the electron density changes with time following switching. It was found that indeed there is a fast (roughly 0.2 picosec) electron transfer between channels $\mathrm{A}$ and $\mathrm{B}$ but a much slower (roughly 3 picosec) redistribution of electrons between the channels that dominate the switching process. This redistribution depends on the impurity concentrations in the channels regions. The switching times for the VMT were 2.0 to 3.0 picosec at $300 \mathrm{~K}$ and about 2.4 picosec at $77 \mathrm{~K}$, significantly shorter than for a more conventional high-electron mobility transistor (over 3.5 picosec at $300 \mathrm{~K}$ and about 3.2 picosec at $77 \mathrm{~K}$ ).

Future semiconductor device modeling will rely even more heavily on supercomputing as shorter length scales involve quantum effects not dominant in today's devices. The reduction in the time and cost between concept and finished product will be a driving force.

\section{We Have Come to Several Conclusions}

Because of its diversity, materials science has evolved into many subspecialties and splintered disciplines. Much progress has been made in understanding the individual aspects of materials behavior under specific environmental and manufacturing conditions. In order to optimize their usage and to exploit, many of these aspects must be integrated and quantitatively described. In general this integration has rarely been achieved, and materials development and applications have been slow endeavors. In applications of materials our inability to precisely quantify materials limitations leads to overdesign, adherence to traditional materials, and "proven" methods of usage, rather than tailored and optimal designs.

The unification of materials science for the pur- pose of improved economic use and for new applications requires supercomputers for explorations and test simulations on shorter time scales and in more cost-effective ways than hitherto practiced. We have demonstrated by the examples in this report that supercomputers are already used for materials research, development, and processing in certain applications and branches of industry. The great economic benefits from supercomputers in materials science and technology are therefore evident but hardly exploited. This underutilization will only become more acute with the rapid advances in supercomputer development unless decisive steps are taken now.

1. Supercomputer simulation has a strong potential to be a powerful, cost-effective link between materials science and engineering, design, and manufacturing. The emerging advanced computers will make possible a new type of materials simulation whose key contribution will be the fostering of interdisciplinary research and development, not only within the materials fields but between these fields and other disciplines. Nowhere is this more important than establishing the bridge between materials, design, and manufacturing technologies.

These methodologies are rapidly developing toward the point where a designer can sit at a graphics terminal and watch the impact of design modifications in real time. Indeed, in some areas these capabilities have already arrived. For silicon chemical vapor deposition, an engineer or technician can continuously change the operating conditions for manufacturing using a minicomputer simulator (a tool based on physical models developed using supercomputing over nearly a decade.). In the automotive industry (where design and tooling cost hundreds of millions of dollars annually) supercomputing aids the design of dies for forming sheet metal for new automobile models. The advanced computations allow physically realistic metal deformation behavior to be applied to forging large, complex parts under production conditions, significantly reducing costs.

As supercomputer and graphics hardware also evolve, we see a time just a few years away when computer simulation of materials and processes 
will play a key role in a majority of U.S. materials technologies.

2. New theoretical methods, algorithms, and approaches for analyzing the individual components of complex materials systems are rapidly evolving. This is enabling methodologies for computer simulation of materials and materials processing to emerge. Fundamental electronic structure calculations can predict the behavior of molecules and solids to such types of accuracies that in many cases computation has become the method of choice over experiment.

Nonetheless, it must not be forgotten that any simulation, however sophisticated, is only as good as its supporting experimental database. Even where theory has led experiment, it is experiment that proves the result. Fortunately, there is a concurrent deepening of this database as computers become more capable.

Supercomputer algorithms now allow the simultaneous solution of hundreds of equations necessary to describe a complex process. Three-dimensional solutions of heat transfer, structural, dynamical, and fluid mechanical equations are possible. These methods may be highly sophisticated, such as by including microstructural evolution during deformation of a metal. Transport calculations in semiconductors can predict electron velocity in high electric fields, properly taking account of the band structure of the solid, leading " to the development of revolutionary electronic devices.

The time is ripe for these more individual efforts to be synthesized into comprehensive computer simulations of materials and materials processes.

3. To exploit opportunities in computational materials science and engineering we must invest heavily in the science underlying the methodologies, in developing software to make these useful to engineers and technicians, and in providing adequate hardware to scientists and engineers at universities and in industry. Fortunately the supercomputer and high-performance graphics hardware needed for routine application of such simulations is continuing to become more powerful and plentiful while dropping in price. The realization of these benefits will require and stimulate the training of a new breed of scientists and engineers, who are equally at home with chemistry, physics, and materials science as well as with modeling and computational methods.

4. For the United States to remain a leader as materials simulation moves out of the laboratory and into the industrial plant, we must act decisively now to stimulate the development of these methodologies and to develop turnkey simulation systems for industrial plants. Although the dawn of this new age for computer simulation in materials processes is just approaching, we can already see arenas where these methodologies are having a major impact.

5. Simulations can reduce design costs and the time from concept to market. We found two industries, in addition to automobile manufacturing, that are using supercomputers to save millions of dollars and months of design time. First, the polymer industry is designing dies by process simulation, resulting in enormous savings relative to the "trial-and-error" approach. Second, the semiconductor industry is turning to computation as the scale of electronic devices decreases. At present, fundamental understanding of these revolutionary devices can only be achieved through simulation. Supercomputing is also being applied to the design of the equipment with which to manufacture semiconductor devices and components.

6. Supercomputers allow us to build hierarchical design tools. The design of chemical vapor deposition equipment is but one example of the growing use of hierarchical design tools among computational scientists and engineers. The hierarchy includes equations and algorithms from different disciplines (i.e., chemistry, physics, materials science, and fluid dynamics) and process that occur over different scales in time and space. A chemical reaction takes place because electrons that rearrange on the scale of angstroms lead to effects on a semiconductor device that may be felt on a scale of centimeters. This is the first time a tool has emerged that makes it possible to integrate complex phenomena from any number of disciplines into a useful, coherent program or code. The modules of the code can be improved as our understanding and the power of computation increase. Furthermore, the designer using the code need not be an expert in the fields of science and engineering represented.

7. Supercomputer simulation is a powerful design tool that can increase U.S. economic competitiveness today. Among the most promising 
areas for further progress are:

- Polymer forming and processing

- Alloy design and processing

- Ceramics design and processing

- Electronic devices

- Electronic device processing equipment

Computer hardware technology is rapidly improving; an order-or-magnitude increase in capability every 5 to 10 years continues to be the norm, with the immeasurable prospects of parallel processing on the horizon. The availability of this evolving state-of-the-art hardware to the engineering and scientific community is essential to the development and continued growth of the algorithms and methodologies that support the application of simulations to new materials technology.

We Llave a Number of Recommendations

1. The committee recommends that focused multidisciplinary research initiatives in selected areas of materials science and engineering be started in order to integrate design and manufacturing with the underpinning research base. Supercomputer simulation of materials phenomena is at a stage where rapid new developments in science and technological applications are possible. Its early uses have been oriented toward well-defined applications and to a large extent have been possible only because of prior investment in long-term fundamental research. Opportunities now exist for simulation to address complex materials phenomena resulting from the interaction of several discrete processes, to provide information that cannot be obtained in any other manner, and to conduct benchmark computations to validate and sometimes guide theoretical modeling, experiment, and the design of new materials. Thus simulation will play a vital role as an adjunct to theory and experimentation. It is essential that suitable leadership, organization, and resources be extended to mobilize and focus our vast national talent on the challenge of designing new materials and materials processes. Some of the initiatives envisioned are:

- Integrated alloy design and synthesis: Simulation of materials microstructure, synthesis, and performance is an achievable objective now in some elementary cases; it is a vital long-range goal for U.S. technology to retain a forefront position in high-value-added materials and devices. One goal would be design of new optoelectronic quantum devices, particularly involving modeling of alloy and composite semiconductor materials.

- Materials processing and manufacturing methods: Substantial progress has already been made in this area. Many opportunities remain to map out operating parameter regimes for producing sound versus defective materials. Areas ripe for development include semi-conductor thin-film deposition and growth associated with integrated circuit technologies, joining methods for metals and ceramics, and forming operations - particularly shaping and molding complex parts.

- Surface and interface phenomena: Examples of promising topics include (a) adhesion - initiation of bonding across interfaces and the effects of impurities, inelastic processes, and defects; and (b) nucleation and growth of thin films - by processes such as molecular beam epitaxy and chemical vapor deposition.

- Atomistic simulation of microstructural evolution: A long-range effort in this area is essential to learn how to perform simulations that involve different time or length scales. Microstructural simulations need atomic-scale information to understand the molecular and chemical structure of defects. Simultaneously, large-scale effects (e.g. millions of dislocations) require shifting attention to the dynamics of defect behavior. Simulations are a powerful means of understanding nonequilibrium effects such as metastable alloy phases and rapid chemical reactions.

2.The committee recommends the creation of a Supercomputing Center for Materials Applications (SCMA). The Center would provide a muchneeded means for moving the results of basic research into turnkey systems suitable for wide use in industry.

- The mission of the Center would be to provide a multidisciplinary environment in which to create software to be transferred to industry to enhance our competitiveness. This must be a "mission-oriented" facility whose management understands the role of the computer as a tool at the Center.

- The Center must be capable of handling proprietary information in order to attract in- 
dustrial funding and to transfer the technology to an interested community. The Center must be able to produce software portable to U.S. industry. Smaller machines and machines with a variety of architectures must therefore be included at the Center for technological transfer purposes.

- The Center should become self-supporting after an initial, properly funded start-up phase.

- The Center should have a permanent professional staff for continuity in addition to an extensive visiting faculty program. Projects aimed at producing software for a given application could bring together a critical mass of talents from around the nation as well as from the site itself. Long- and short-term projects would both be addressed. Such a center should also provide access to novel computer architectures, as required.

- The Center should be advantageously located within an existing supercomputer center. There are significant economies of scale in hardware to be gained by such a colocation.

- The Center should be advantageously located within an organization accustomed to an applications-oriented, multidisciplinary approach to supercomputing.

- The Center should be advantageously located near broadly based expertise in materials science and engineering. The daily interactions among a diverse scientific staff (including materials scientists, physicists, chemists, engineers, numerical analysts, and applied mathematicians) are of most value.

3. The committee recommends that supercomputer centers be kept at the state of the art. The momentum begun with the creation of the national supercomputer centers (for example, the NSF initiative) should be accelerated by a longterm commitment to increase the availability of computer resources to a broad scientific community. Access to state-of-the-art mainframes must be included as soon as they become available. Supercomputer technology is moving ahead at a rate that will render them obsolete in a few short years. Hence the retooling of these centers must be carefully coordinated: These are not one-time investments - they must be continually upgraded.
- The capacity of these centers needs to be expanded as well, but we again caution against the misconception that a computer center's power be measured by the total number of arithmetic operations it can perform (capacity). What really counts is how many operations can be applied to a single problem at the same time (capability), which is measured by the power of its largest (fastest) mainframe.

- The centers must include a commitment to upgrade their internal communications networks as well as the network with the external user community. High-bandwidth communications are necessary for interactive use and to take proper advantage of essential graphics.

4. The committee recommends the formation of multidisciplinary materials simulation groups (MSGs). The formation of MSGs (of several faculty spread over several departments) will create a synergy of materials scientists with computer scientists with computer scientists, computational mathematicians, and experts in visualization. They will generally work closely with the SCMA. New ideas in technology will require advanced computational strategies, new algorithms, and unprecedented capability to analyze and visualize results. Such groups and capabilities need to be developed and brought toward more effective solution of important scientific and technological materials research problems.

5. The committee recommends that the infrastructure of local computing associated with advanced simulation be strengthened. Solving current materials problems through simulation requires the capability of the best mainframes. Yet the trial of new algorithms, development of codes, and display of results are better accomplished in a local environment. Thus there needs to be recognition that the acquisition of advanced local computing capabilities is an important ingredient in the overall structure of advanced computation.

6. These initiatives should be implemented in a manner balanced to preserve the research strengths that are central to the success of materials engineering and processing in the future. We can today simulate several materials processes only because of the long-term fundamental research investment in the past. 